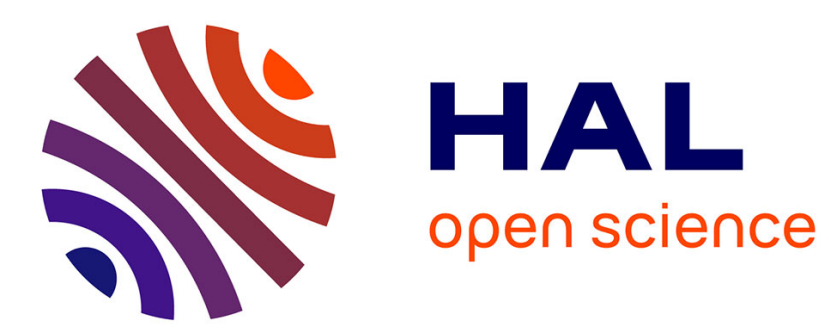

\title{
Surface self-diffusion of silicon during high temperature annealing
}

Pablo E. Acosta-Alba, Oleg Kononchuk, Christophe Gourdel, Alain Claverie

\section{To cite this version:}

Pablo E. Acosta-Alba, Oleg Kononchuk, Christophe Gourdel, Alain Claverie. Surface self-diffusion of silicon during high temperature annealing. Journal of Applied Physics, 2014, 115 (13), pp.134903 333. 10.1063/1.4870476 . hal-01719499

\section{HAL Id: hal-01719499 \\ https://hal.science/hal-01719499}

Submitted on 28 Feb 2018

HAL is a multi-disciplinary open access archive for the deposit and dissemination of scientific research documents, whether they are published or not. The documents may come from teaching and research institutions in France or abroad, or from public or private research centers.
L'archive ouverte pluridisciplinaire HAL, est destinée au dépôt et à la diffusion de documents scientifiques de niveau recherche, publiés ou non, émanant des établissements d'enseignement et de recherche français ou étrangers, des laboratoires publics ou privés. 


\section{Surface self-diffusion of silicon during high temperature annealing}

Pablo E. Acosta-Alba, Oleg Kononchuk, Christophe Gourdel, and Alain Claverie

Citation: Journal of Applied Physics 115, 134903 (2014); doi: 10.1063/1.4870476

View online: https://doi.org/10.1063/1.4870476

View Table of Contents: http://aip.scitation.org/toc/jap/115/13

Published by the American Institute of Physics

\section{Articles you may be interested in}

Shape transformation of silicon trenches during hydrogen annealing

Journal of Vacuum Science \& Technology A: Vacuum, Surfaces, and Films 21, 1279 (2003); 10.1116/1.1586278

Theory of Thermal Grooving

Journal of Applied Physics 28, 333 (1957); 10.1063/1.1722742

Hydrogen pressure dependence of trench corner rounding during hydrogen annealing

Journal of Vacuum Science \& Technology A: Vacuum, Surfaces, and Films 22, 1406 (2004); 10.1116/1.1760752

Growth of native oxide on a silicon surface

Journal of Applied Physics 68, 1272 (1990); 10.1063/1.347181

Formation of periodic step and terrace structure on $\mathrm{Si}(100)$ surface during annealing in hydrogen diluted with inert gas

Journal of Vacuum Science \& Technology A: Vacuum, Surfaces, and Films 16, 1775 (1998); 10.1116/1.581301

Silicon surface passivation by hydrogen termination: A comparative study of preparation methods Journal of Applied Physics 66, 419 (1989); 10.1063/1.343839

\section{Scilight}

Sharp, quick summaries illuminating the latest physics research

\section{Sign up for FREE!}

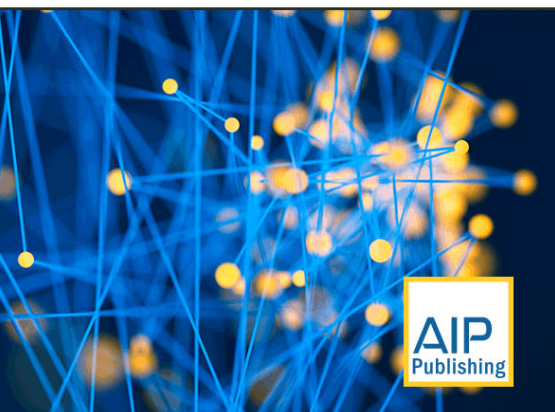




\title{
Surface self-diffusion of silicon during high temperature annealing
}

\author{
Pablo E. Acosta-Alba, ${ }^{1,2, a)}$ Oleg Kononchuk, ${ }^{2}$ Christophe Gourdel, ${ }^{2}$ and Alain Claverie ${ }^{1}$ \\ ${ }^{1}$ CEMES-CNRS and Université de Toulouse, 29 Rue Jeanne Marvig, BP 94347, 31055 Toulouse Cedex 4, \\ France \\ ${ }^{2}$ Corporate R\&D, SOITEC, Parc Technologique des Fontaines, Chemin des Franques, 38190 Bernin, France
}

(Received 2 December 2013; accepted 24 March 2014; published online 3 April 2014)

\begin{abstract}
The atomic-scale mechanisms driving thermally activated self-diffusion on silicon surfaces are investigated by atomic force microscopy. The evolution of surface topography is quantified over a large spatial bandwidth by means of the Power Spectral Density functions. We propose a parametric model, based on the Mullins-Herring (M-H) diffusion equation, to describe the evolution of the surface topography of silicon during thermal annealing. Usually, a stochastic term is introduced into the $\mathrm{M}-\mathrm{H}$ model in order to describe intrinsic random fluctuations of the system. In this work, we add two stochastic terms describing the surface thermal fluctuations and the oxidation-evaporation phenomenon. Using this extended model, surface evolution during thermal annealing in reducing atmosphere can be predicted for temperatures above the roughening transition. A very good agreement between experimental and theoretical data describing roughness evolution and self-diffusion phenomenon is obtained. The physical origin and time-evolution of these stochastic terms are discussed. Finally, using this model, we explore the limitations of the smoothening of the silicon surfaces by rapid thermal annealing. (C) 2014 AIP Publishing LLC.
\end{abstract}

[http://dx.doi.org/10.1063/1.4870476]

\section{INTRODUCTION}

Mastering the thickness uniformity of the top silicon layer is a real challenge for the new generation CMOS technology based on Fully Depleted Silicon-On-Insulator (FDSOI) wafers. These thickness variations have to be tightly controlled over a wide spatial bandwidth, from the transistor scale to the wafer scale. ${ }^{1,2}$ In particular, topological variations at high spatial frequencies are critical. Indeed, it has been shown that for FD-SOI wafers, thickness and topological variations of the top layer are similar all over a large spatial scale (from few nanometers up to few microns). ${ }^{3}$ Hence, thickness variations can be directly estimated only by measuring the topological variations over this spatial range.

Surface morphology can be modified through the transport of material based on different phenomena: plastic deformation, evaporation, condensation, volume, and surface diffusions. The characteristic time $\tau$ associated to the surface evolution depends on the system dimension $\mathrm{L}$, according to the scaling law $\tau \sim L^{z}$, where $z$ is named the dynamic exponent and is usually written as $z=\alpha / \beta, \alpha$ being the roughness exponent and $\beta$ being the growth exponent which, respectively, describes the evolution of the Root-Mean-Square (RMS)-Roughness as a function of the system dimensions and the RMS-roughness evolution with time. ${ }^{4}$ A maximum value of the dynamic exponent $(z=4)$ is obtained for surface self-diffusion. This reflects that surface self-diffusion is the most efficient mechanism to reduce surface roughness. Hence, most of techniques used to reduce surface topography variations at high spatial frequencies (up to few microns length) are based on surface self-diffusion. One of such techniques is high temperature annealing in inert or reducing

a)pablo-eduardo.acosta-alba@soitec.com atmosphere. Thus, the understanding of the atomic-scale mechanisms describing surface self-diffusion on silicon, at high temperatures is of great interest. Many authors have experimentally studied surface self-diffusion through the shape transformation of periodic superficial silicon structures during annealing at high temperature (above the silicon roughening temperature)..$^{5-7}$

At high temperatures, the surface tends to reduce its energy by minimizing surface area using self-diffusion of adatoms. Then, the evolution of surface topography by mass transport can be quantitatively described by a surface selfdiffusion mechanism. This process was macroscopically described by Mullins in the case of surfaces of low curvature and isotropic surface energy using the so-called MullinsHerring (M-H) equation. ${ }^{4,6,8,9}$

In this work, we study the evolution of the surface topography of the top silicon layer of SOI wafers during rapid thermal annealing (RTA) at high temperature. Annealed surfaces are analysed by means of the power spectral density (PSD) functions. A parametric model describing the spectral evolution of the silicon surface topography is proposed.

\section{METHODS AND EXPERIMENTAL PROCEDURE}

Silicon surfaces can undergo a roughening transition from a faceted to a rough surface above the roughening temperature $\left(T_{R}\right)$, losing the anisotropic nature of self-diffusion. For example, for $\mathrm{Si}(001) T_{R}$ is approximately $1050{ }^{\circ} \mathrm{C} .{ }^{10-12}$ The thermal treatments investigated in this work are done at high enough temperatures to consider diffusion to be isotropic. Considering typical durations of a RTA process (few seconds), thermal annealing above $T_{R}$ leads to diffusion lengths $\left(\lambda_{D}=\sqrt{D_{s} t}\right.$ where $D_{s}$ is the surface diffusion coefficient which characterizes the surface diffusion rate of 
adatoms for a given temperature) of at least few micrometers. ${ }^{7,13}$ Thus, in order to observe the evolution of surface topography during RTA at high temperatures, it is important to use a starting material whose initial topography shows high vertical variations at those characteristic lengths.

In the Smart CutTM process, the fracture of silicon induced by ion implantation is used to transfer thin silicon films. This fracture leads to a surface showing fractal characteristics with a low roughness exponent $(\alpha)$ equal to $0.4 \pm 0.1 .^{3}$ Moreover, the evolution of hydrogenated defects induced by implantation, responsible for the silicon fracture, leads to the formation of micro-cracks with size-distributions in the range of a few micrometers which determine the roughness of the post-fracture silicon surfaces. ${ }^{14}$ It makes post-fracture SOI surfaces a very suitable object to study the impact of rapid thermal annealing on the surface topography.

A set of $300 \mathrm{~mm}$-in-diameter SOI wafers was fabricated by the Smart CutTM process. All finishing steps were omitted in order to obtain fractured-like surfaces. Then, wafers were annealed in a mixture of hydrogen and argon ambient (20\% and $80 \%$, respectively) at a pressure of 760 Torr. The $\mathrm{O}_{2}$ and $\mathrm{H}_{2} \mathrm{O}$ concentrations in the mixture gas used in the process are controlled to be less than $0.1 \mathrm{ppb}$ and $0.2 \mathrm{ppb}$. The annealing temperatures were $1050{ }^{\circ} \mathrm{C}, 1100^{\circ} \mathrm{C}, 1150{ }^{\circ} \mathrm{C}, 1200^{\circ} \mathrm{C}$, and $1250{ }^{\circ} \mathrm{C}$, for six different durations, from $0 \mathrm{~s}$ to $90 \mathrm{~s}$ plateau. In our experiment, the process time corresponds to the time actually passed at the target temperature. Indeed, the equivalent thermal budget of the $0 \mathrm{~s}$ plateau, corresponds to that of ramp-up to the target temperature directly followed by ramp-down. The wafers were fed into the annealing chamber at $700^{\circ} \mathrm{C}$, the temperature ramp-up and ramp-down were both $50{ }^{\circ} \mathrm{C} \mathrm{s}^{-1}$. Experiments were performed in a rapid thermal processing system Helios XP from Mattson.

\section{RESULTS AND DISCUSSION}

The surface roughness of each annealed wafer was characterized by means of atomic force microscopy (AFM). In order to cover the spatial bandwidth of interest (from $0.067 \mu \mathrm{m}^{-1}$ to $128 \mu \mathrm{m}^{-1}$ ) multiple images with sizes $2 \times$ $2,10 \times 10$, and $30 \times 30 \mu \mathrm{m}^{2} \quad(512 \times 512$ pixels $)$ were recorded. Figures 1-3 show typical AFM images in $2 \times 2$ and $30 \times 30 \mu \mathrm{m}^{2}$ obtained from the samples before and after RTA process. In Figure 1, it can be observed that before RTA the fractured silicon surface presents a microroughness with a high vertical amplitude. Moreover, profiles in the figure evidence that topography variations present characteristic lengths between $100 \mathrm{~nm}$ and $4 \mu \mathrm{m}$. These characteristic lengths come from the evolution of implantinduced hydrogenated defects as explained above.

Figure 2 shows the roughness of fractured silicon wafer after " $0 \mathrm{~s}$ " RTA process at $1200^{\circ} \mathrm{C}$ (ramp up to $1200^{\circ} \mathrm{C}$ followed by the ramp down). We note that the amplitude of vertical variations of the surface is considerably decreased while the lower characteristic length increases up to $0.5 \mu \mathrm{m}$. Figure 3 evidences a larger decrease of amplitude of vertical variations of the surface after $90 \mathrm{~s}$ RTA process, while its characteristic length increases up to $3 \mu \mathrm{m}$.

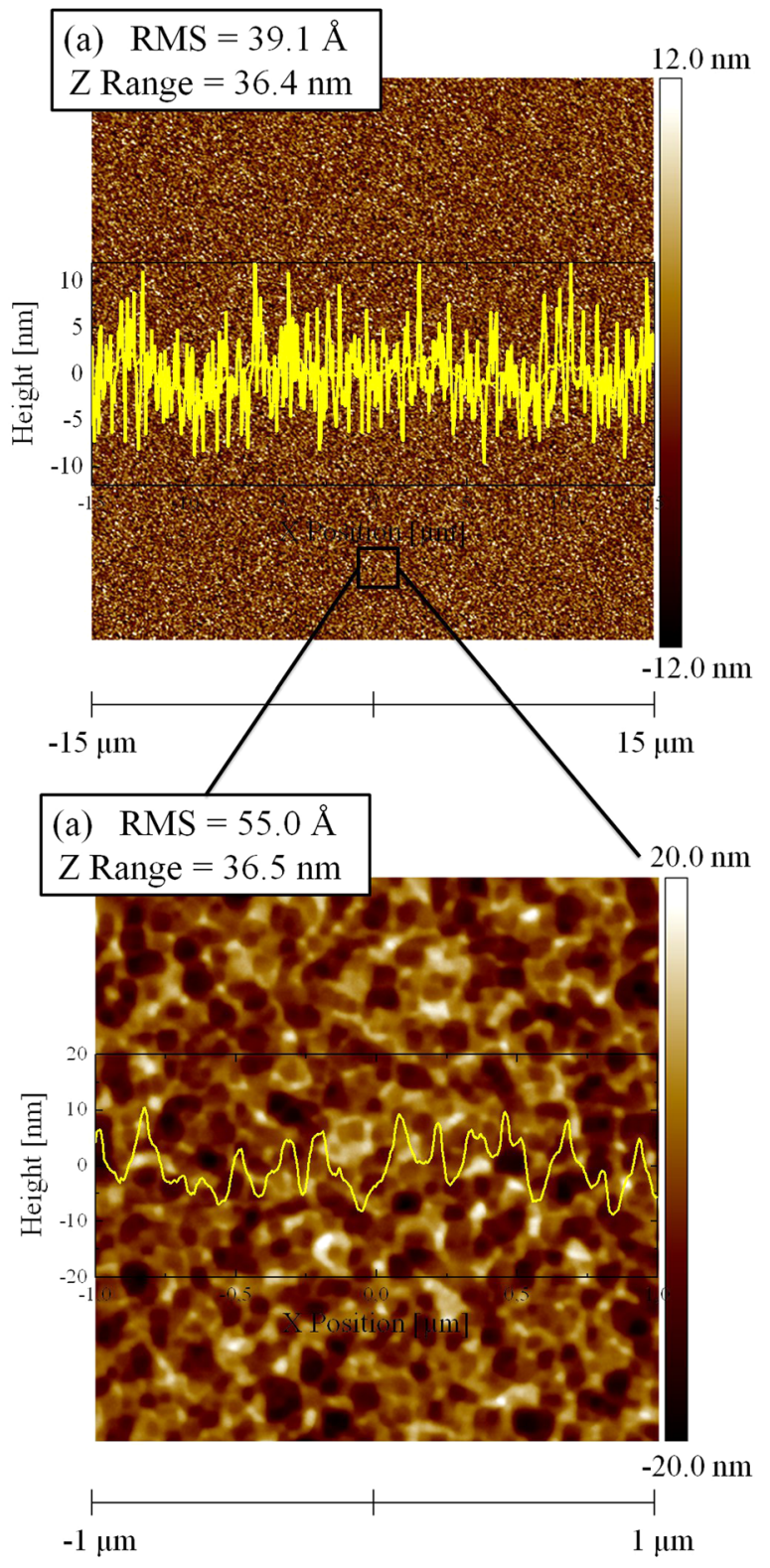

FIG. 1. Images obtained by AFM of the surface roughness before any thermal annealing (just after surface preparation). Scan sizes (a) $30 \times 30 \mu \mathrm{m}^{2}$ and (b) $2 \times 2 \mu \mathrm{m}^{2}$ (both sizes with $512 \times 512$ pixels). Profiles extracted from the AFM images are plotted and show the characteristic lengths of each images.

The topography of a surface can be described by the space-time vector $(h(\vec{x}, t))$, known as the local height function of the surface. The roughness of a surface is usually described by its RMS value. RMS is the standard deviation of $h(\vec{x}, t)$ which is composed of discrete points describing the local height surface. This well-known quantity provides a measurement for the overall roughness of the surface regardless of its spatial distribution and relevant lengths. Figure 4 shows the variation of the RMS-Roughness values of surfaces as a function of the annealing duration, at $1200^{\circ} \mathrm{C}$. It can be observed that the RMS-Roughness decreases as a power function of the annealing time with a growth exponent $\beta=-0.25 \pm 0.02$ This value is in perfect agreement with self-diffusion theory which predicts an exponent equal to $\beta=-1 / 4 .^{4,8,9}$ 


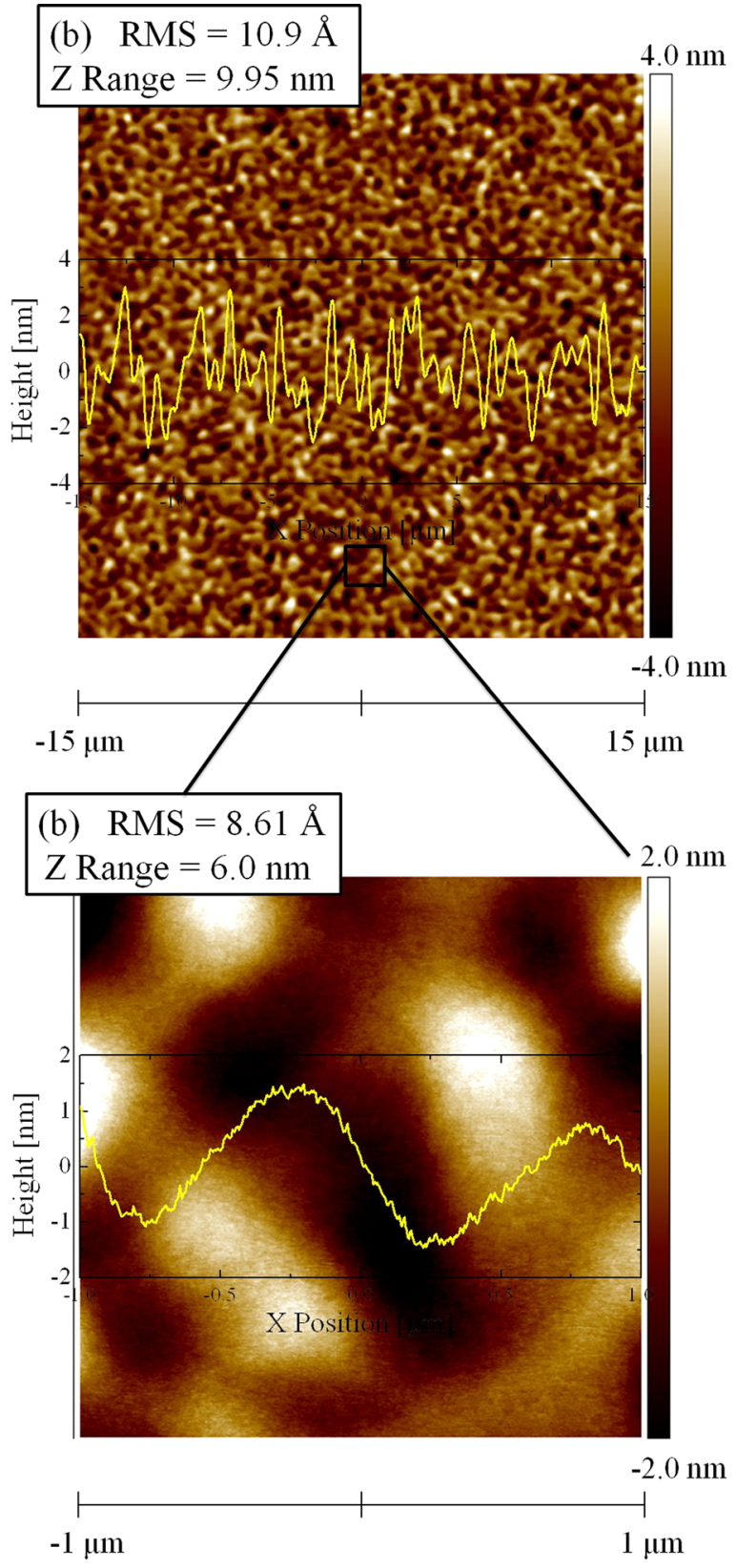

FIG. 2. Images obtained by AFM of the surface roughness after thermal annealing corresponding to " $0 \mathrm{~s}$ "-plateau at $1200^{\circ} \mathrm{C}$. the thermal budget is equivalent to the ramp-up to $1200^{\circ} \mathrm{C}$ followed by the ramp-down. Scan sizes (a) $30 \times 30 \mu \mathrm{m}^{2}$ and (b) $2 \times 2 \mu \mathrm{m}^{2}$ (both sizes with $512 \times 512$ pixels). Profiles extracted from the AFM images are plotted and show the characteristic lengths of each image.

Furthermore, to accurately quantify the contribution of each spatial frequency to the measured roughness, the PSD function can be used. Indeed, PSD function provides a complete statistical description of surfaces since it represents a frequency decomposition of the power of a signal. ${ }^{3}$ Hence, it is more suitable than RMS value for characterization of the roughness evolution over a large range of spatial frequencies (from $0.067 \mu \mathrm{m}^{-1}$ to $128 \mu \mathrm{m}^{-1}$ ).

Figure 5 shows the time evolution of the twodimensional PSD (2D-PSD) function obtained from AFM $30 \times 30 \mu \mathrm{m}^{2}$ images of the annealed surfaces (see experimental data in Fig. 5). We can observe two characteristic

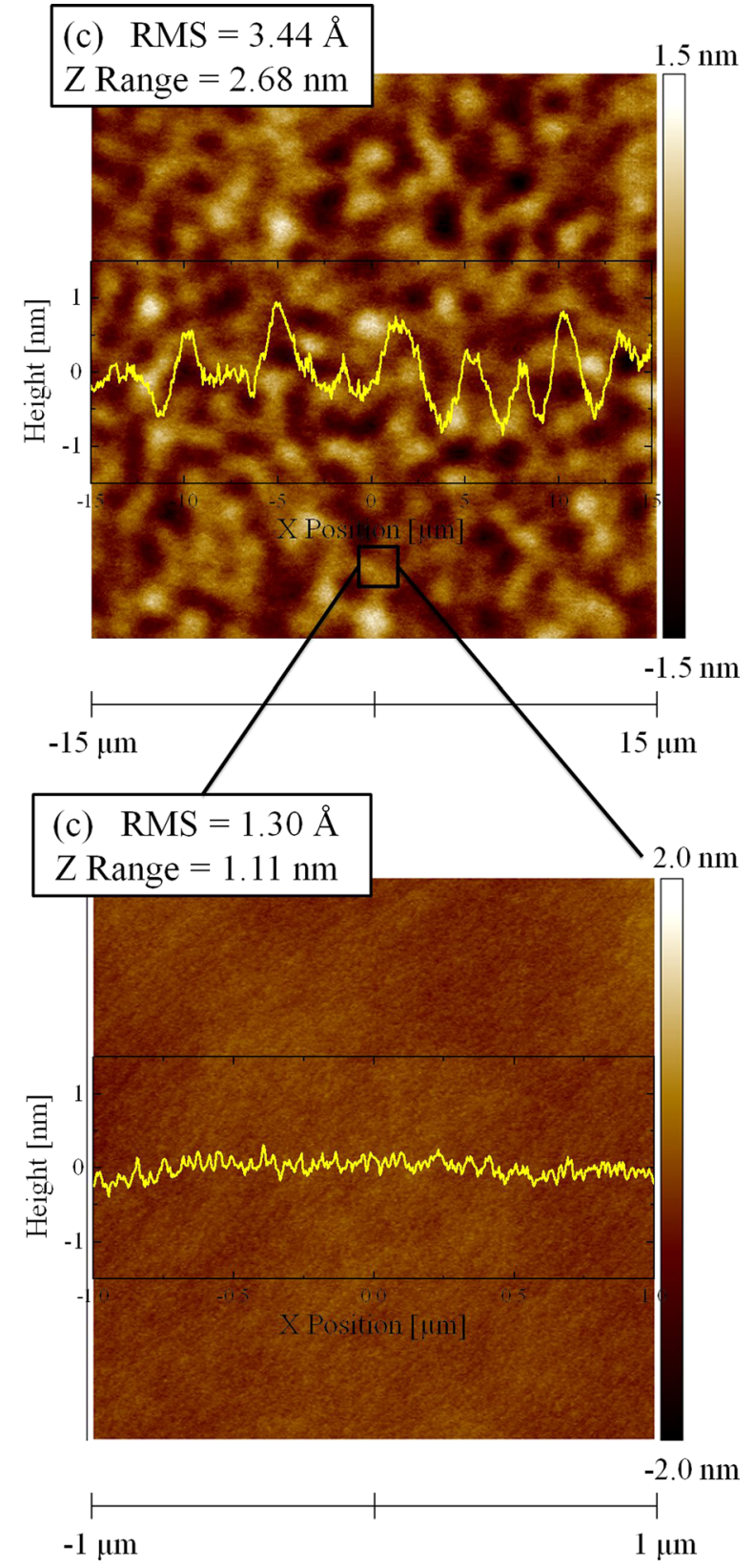

FIG. 3. Images obtained by AFM of the surface roughness after 90 s-plateau annealing at $1200^{\circ} \mathrm{C}$. Scan sizes (a) $30 \times 30 \mu \mathrm{m}^{2}$ and (b) $2 \times 2 \mu \mathrm{m}^{2}$ (both sizes with $512 \times 512$ pixels). Profiles extracted from the AFM images are plotted and show the characteristic lengths of each image.

cut-off frequencies; the first one (from $0.3 \mu \mathrm{m}^{-1}$ up to $\left.1 \mu \mathrm{m}^{-1}\right)$, which will be named diffusion-frequency $\left(f_{D}\right)$, corresponds to the lowest spatial frequency impacted by the thermal smoothening. In fact, for lower spatial frequencies, the amplitude of the 2D-PSD function remains unchanged implying that surface variations whose characteristic length corresponds to spatial frequencies lower than diffusionfrequency are not altered by the thermal annealing under our experimental conditions. Indeed, $f_{D}$ corresponds to the frontier between this unchanged region and another region in which 2D-PSD amplitude decreases rapidly (see regions I and II in Fig. 6). The value of $f_{D}$ depends on the annealing duration and corresponds to the diffusion length of the system for a given thermal budget. 


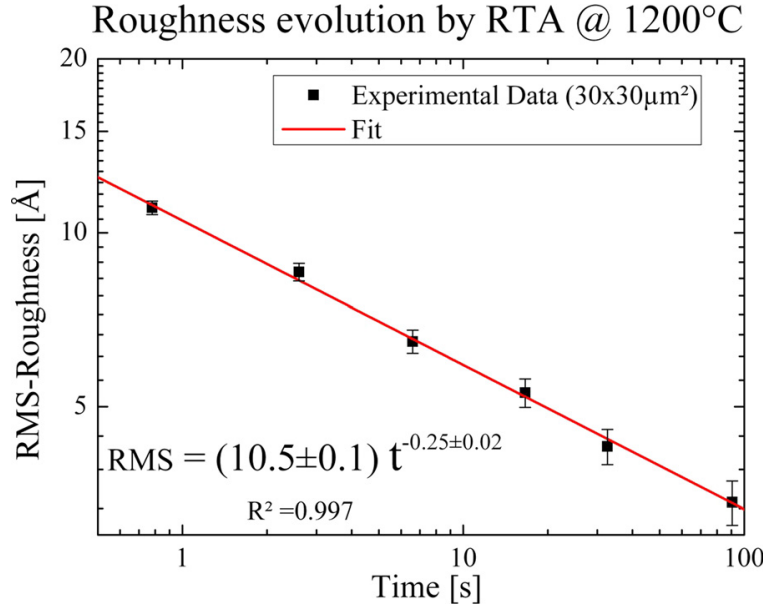

FIG. 4. Evolution of the RMS surface roughness as a function of annealing duration. RMS values are obtained from $30 \times 30 \mu \mathrm{m}^{2}$ AFM images. The RMS value decreases as a power function of the annealing time with an exponent equal to $-0.25 \pm 0.02$ which is in perfect agreement with self-diffusion theory $(\beta=1 / 4))^{4,8,9}$

The second cut-off frequency (from $0.7 \mu \mathrm{m}^{-1}$ up to $\left.2.5 \mu \mathrm{m}^{-1}\right)$, which we will name noise-frequency $\left(f_{N}\right)$ below, shows the limit between two regions (see region II and III in Fig. 6) with different diffusion behaviours. Indeed, for frequencies lower than the noise-frequency (and higher than the diffusion frequency, see region II in Fig. 6), we observe a progressive reduction of the amplitude of the 2D-PSD function. The observed roughness exponent $\alpha$ (calculated from the slope of the 2D-PSD function) in this region, is equal to $1.5 \pm 0.1$ and agrees well with a surface diffusion phenomenon which belongs to the MBE (Molecular Beam Epitaxy) universality scaling class. ${ }^{4,16}$ Indeed, this scaling class predicts scaling behaviour with exponents $\alpha=3 / 2, \beta=3 / 8$ and $z=4 .{ }^{17,18}$ For frequencies higher than $f_{N}$, the amplitude of the 2D-PSD function of surfaces after thermal annealing, dramatically decreases compared to the amplitude of initial 2D-PSD function. It means that surface variations with very small characteristic lengths are considerably smoothened during thermal annealing. However, the observed roughness exponent is much smaller than the one corresponding to a surface self-diffusion phenomenon (see region III in Fig. 6).

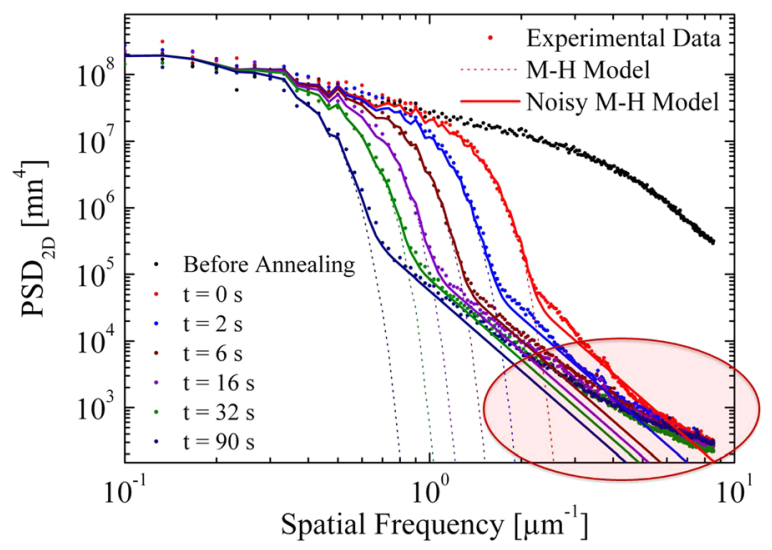

FIG. 5. 2D-PSD spectra of silicon surfaces annealed by RTA at $1200^{\circ} \mathrm{C}$ during different times. Experimental data (points) are compared to the $\mathrm{M}-\mathrm{H}$ model (dashes) and to the quenched M-H model (solid lines).

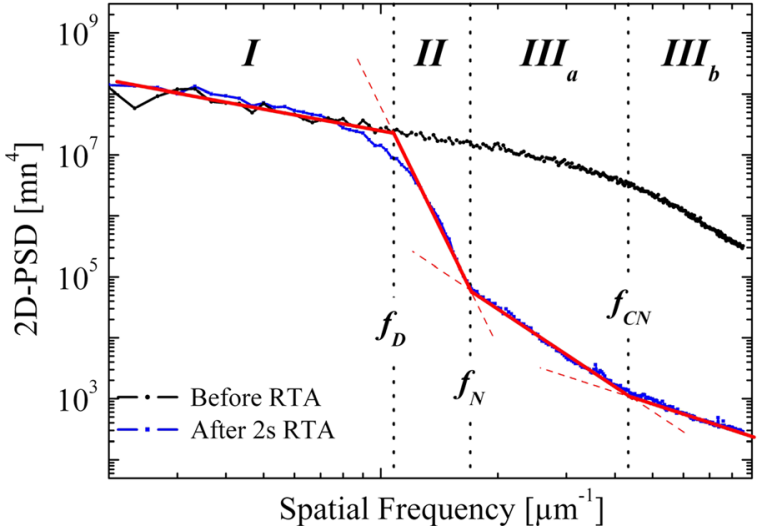

FIG. 6. Schematic representation of the 2D-PSD function of annealed surface $\left(2 \mathrm{~s}\right.$ at $\left.1200^{\circ} \mathrm{C}\right)$. Four regions with different scaling behaviour are evidenced. These regions are separated by: Diffusion-frequency $\left(f_{D}\right)$, noisefrequency $\left(f_{N}\right)$ and conservative noise frequency $\left(f_{C N}\right)$.

This suggests that for spatial frequencies higher than $f_{N}$, additional thermally activated phenomena take place at the surface. The possible origin of such phenomena is discussed below.

Surface self-diffusion is described by the M-H model which macroscopically describes the evolution of the height function by surface self-diffusion of adatoms. The $\mathrm{M}-\mathrm{H}$ model is expressed by the following, M-H equation: ${ }^{17}$

$$
\frac{\partial h(\vec{x}, t)}{\partial t}=-\nu_{4} \nabla^{4}(h(\vec{x}, t)),
$$

where $\nu_{4}=D_{s} \gamma_{s} v \Omega^{2} / k_{B} T$, is directly related to the surface diffusion coefficient $\left(D_{s}\right),(T)$ is the annealing temperature, $\gamma_{s}$ is the surface energy, $v$ is the atomic density, $\Omega$ is the molecular volume of silicon, and $k_{B}$ is the Boltzmann constant.

Even if M-H equation (Eq. (1)) is deterministic, there is an inherent randomness in the system. Indeed, surface selfdiffusion is defined in terms of adatoms jumping between adjacent adsorption sites on the surface and thus is a probabilistic process. This randomness can be described by a stochastic term $(\eta(\vec{x}, t)$ added to the right-hand side of the M-H equation ${ }^{4,16}$ (see Eq. (2)).

$$
\frac{\partial h(\vec{x}, t)}{\partial t}=-\nu_{4} \nabla^{4}(h(\vec{x}, t))+\eta(\vec{x}, t) .
$$

$\eta(\vec{x}, t)$ is a non-conservative noise ( $\mathrm{NCN})$ which is uncorrelated in space and time (Eq. (2) is commonly called the noisy-MH equation). In case of RTA, this term reflects the surface evolution due to the oxidation-evaporation of silicon, which is considered to take place randomly at the silicon surface. The space-average of this NCN must be zero $(\langle\eta(\vec{x}, t)\rangle=0)$. The correlator of the NCN can be written as $\left\langle\eta(\vec{x}, t) \eta\left(\vec{x}^{\prime}, t^{\prime}\right)\right\rangle=2 \Delta_{N C N} \delta^{d}\left(\vec{x}-\vec{x}^{\prime}\right) \delta^{d}\left(t-t^{\prime}\right){ }^{4,16}$

Figure 5 shows a comparison between the 2D-PSD functions obtained from the experimental data (points), those from the simple M-H model (dashes) and those from the noisy M-H model (solid lines). We can note that the experimental 2D-PSD functions are well-approximated by both, simple and noisy M-H models, for spatial frequencies lower than $f_{N}$. Nevertheless, for spatial frequencies higher than $f_{N}$, 
the amplitude of the 2D-PSD functions obtained from a simple M-H model, decreases too fast and does not describe the evolution of the annealed surfaces. Whereas, the noisy M-H model accurately describes the evolution of the amplitude of the 2D-PSD functions over the whole spatial bandwidth, for annealing durations up to $2 \mathrm{~s}$. However, it can be noted that the noisy M-H model does not describe the 2D-PSD functions at high frequencies for annealing duration larger than $2 \mathrm{~s}$. This suggests that the NCN term introduced in the noisy $\mathrm{M}-\mathrm{H}$ model well-described the thermal smoothening process at the early stages of the RTA, but not for longer times. Then, it is necessary to further investigate the smoothening behaviour at frequencies higher than $f_{N}$.

Figure 7 shows a zoom on the high frequencies domain (higher than $f_{N}$ ) of the 2D-PSD function of a surface annealed at $1200^{\circ} \mathrm{C}$ during $2 \mathrm{~s}$. It can be observed that the 2D-PSD function has two different slopes over this spatial frequency range, separated by a characteristic spatial frequency $\left(f_{C N}\right)$ which will be named conservative-noise frequency. This suggests that two different phenomena drive the surface evolution at such high frequencies. The values of the slope of the 2D-PSD functions are equal to -4 for frequencies lower than $f_{C N}$ (see region a in Fig. 7) and equal to 2 for frequencies higher than $f_{C N}$ (see region b in Fig. 7). These slopes can be related to non-conservative and conservative processes, respectively. ${ }^{17,19}$

Thus, to describe the evolution of the full 2D-PSD spectrum, we propose to introduce a second stochastic term into the noisy M-H diffusion equation (Eq. (2)). The proposed model is described by the following equation:

$$
\frac{\partial h(\vec{x}, t)}{\partial t}=-\nu_{4} \nabla^{4}(h(\vec{x}, t))+\eta(\vec{x}, t)+\eta_{d}(\vec{x}, t),
$$

where the stochastic term $\eta_{d}(\vec{x}, t)$ defines a mass conservative noise $(\mathrm{CN})$ in the system. Indeed, thermal fluctuations change the height of the surface at a given position (by moving adatoms into or away from that position). Every increase (decrease) of the surface height in a particular position is accompanied by the opposite in another nearby position on the surface. Therefore, the integral of the $\mathrm{CN}$ over the surface

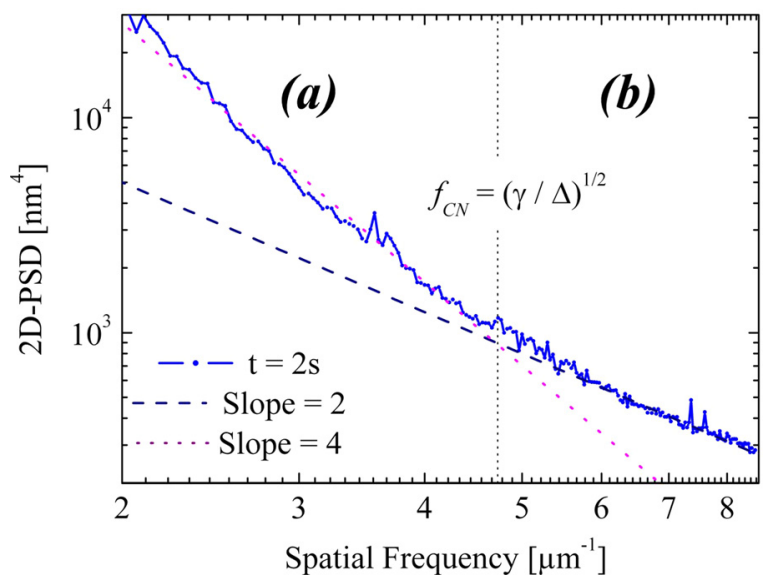

FIG. 7. High frequency range of the 2D-PSD function of annealed surfaces ( $2 \mathrm{~s}$ and $16 \mathrm{~s}$ at $1200^{\circ} \mathrm{C}$ ). Two scaling regimes are evidenced, separated by a characteristic frequency $\left(f_{c}\right)$. must remain zero at every time. It can be easily verified that any noise term with zero-average $\left(\left\langle\eta_{d}(\vec{x}, t)\right\rangle=0\right)$ and with correlator $\left\langle\eta_{d}(\vec{x}, t) \eta_{d}\left(\vec{x}^{\prime}, t^{\prime}\right)\right\rangle=2 \gamma_{C N} \nabla^{2} \delta^{d}\left(\vec{x}-\vec{x}^{\prime}\right) \delta^{d}\left(t-t^{\prime}\right)$ satisfies the requirement of mass conservation. In conclusion, the added $\mathrm{CN}$ term describes the evolution of the surface due to the thermal fluctuation of the adatoms on the silicon surface.

The following rescaling conversion can be applied in order to calculate a characteristic length: $\vec{x} \rightarrow b \vec{x}$ and $t \rightarrow b^{z t}$. We obtain $\eta(\vec{x}, t) \rightarrow \Delta^{1 / 2} \eta(\vec{x}, t) b^{-(d+z) / 2}$ and $\eta_{d}(\vec{x}, t) \rightarrow \gamma_{d}^{1 / 2} \eta_{d}(\vec{x}, t) b^{-(d+z+2) / 2}{ }^{4}$ Then, it is easy to find the characteristic length scale given by

$$
L_{C N}=\left(\frac{\gamma_{C N}}{\Delta_{N C N}}\right)^{2} .
$$

The characteristic frequency $\left(f_{C N}=1 / L_{C N}\right)$ separates two scaling regimes describing two different phenomena. Indeed, for $f \gg f_{C N}\left(\right.$ e.i. $\left.L \ll L_{C N}\right)$, thermal fluctuations (conservative noise) dominate surface evolution, while for $f \ll f_{C N}$ (i.e., $L \gg L_{2}$ ), the deposition/evaporation noise dominates. It has been observed that $f_{C N}$ increases with the annealing duration (see high frequencies domain in Fig. 5). It means that the NCN contribution to the 2D-PSD amplitude decreases with time.

The solution of Eq. (3) in Fourier space can be written as

$$
\begin{aligned}
F T[h(\vec{x}, t)]_{t}= & F T[h(\vec{x}, t)]_{t=0} e^{-\nu_{4} f^{4} t} \\
& +F T[\eta(\vec{x}, t)]+F T\left[\eta_{d}(\vec{x}, t)\right] .
\end{aligned}
$$

It gives the explicit form of 2D-PSD, describing the time-evolution of the surface height as a function of the spatial frequency (Eq. (6)).

$$
\begin{aligned}
\operatorname{PSD}(h(\vec{x}, t))_{t}= & P S D(h(\vec{x}, t))_{t=0} e^{-2 \nu_{4} f^{4} t} \\
& +\frac{\Delta}{\left((2 \pi)^{4} \nu_{4} f^{4}\right)\left(1-e^{-2 \nu_{4} f^{4} t}\right)} \\
& +\frac{\gamma}{\left((2 \pi)^{4} \nu_{4} f^{2}\right)\left(1-e^{\left.-2 \nu_{4} f^{4} t\right)}\right.},
\end{aligned}
$$

where $\Delta=(\Delta x)^{2} \Delta_{N C N}$ and $\gamma=(\Delta x)^{2} \gamma_{C N}$. $\Delta x$ is the sampling period of the Fourier transform and is used as a normalization term for the 2D-PSD functions.

Experimental 2D-PSD functions have been fitted, for each temperature and time by Eq. (6) using three fitting parameters $D_{s}, \Delta$, and $\gamma$. By fitting the experimental curves with the simple $\mathrm{M}-\mathrm{H}$ model over the low frequencies range of 2D-PSD function (frequencies lower than $f_{N}$ ), we can unambiguously obtain the value of $D_{s}$ coefficient for each temperature. Then, using a constant $D_{s}$ value for each temperature, the time evolution of the 2D-PSD function can be fitted varying $\Delta_{N C N}$ and $\gamma_{C N}$ as parameters.

Figure 8 shows the comparison between the experimental 2D-PSD functions and the curves obtained by the model proposed in this work. It can be observed that this model accurately describes the time-evolution of the silicon 


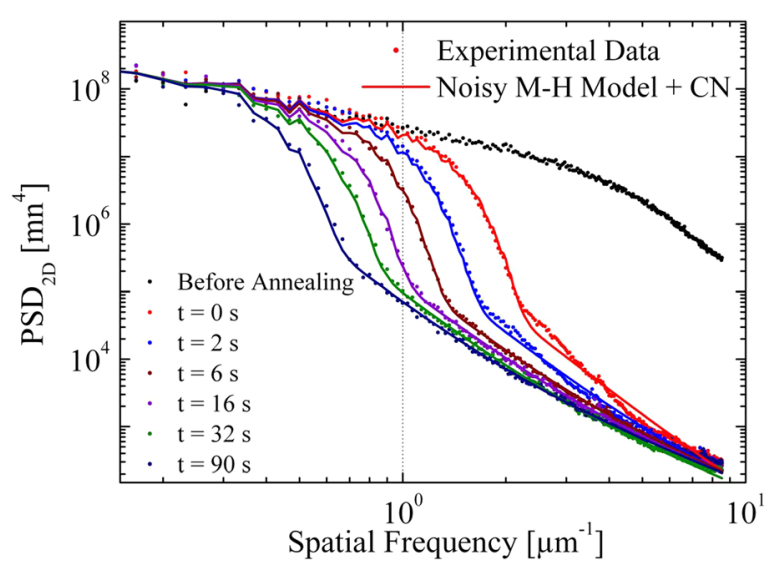

FIG. 8. 2D-PSD functions of surfaces of SOI wafers annealed by RTA at $1200^{\circ} \mathrm{C}$ for different times. Experimental data (points) are compared to the theoretical $\mathrm{M}-\mathrm{H}$ model (dashes) and to the new proposed model (solid lines).

surfaces during RTA for any process duration. It is found that the thermal budget of the ramp-up together with the ramp-down, is equivalent to $0.7 \mathrm{~s}$ at $1200^{\circ} \mathrm{C}$. Indeed, smoothening of silicon surfaces through self-diffusion mechanisms can be observed at low temperatures (above $\left.800^{\circ} \mathrm{C}\right) .{ }^{15}$ Hence, the thermal evolution of the surfaces during the increasing and decreasing temperature can be seen as the evolution at the target temperature during this equivalent time $\left(0.7 \mathrm{~s}\right.$ at $\left.1200^{\circ} \mathrm{C}\right)$.

It is also remarkable that the model well-describes the evolution of the 2D-PSD functions over the whole measured spectrum. Thus, we have set up a predictive model able to describe the evolution of the surface topography of silicon during rapid thermal annealing for any temperature and any duration.

Figure 9 shows the temperature dependence of the silicon surface diffusivity as extracted from our experiments. The obtained $D_{s}$ follows an Arrhenius type law with an activation energy of $E a=2.5 \pm 0.1 \mathrm{eV}$ and a pre-exponential factor of $0.3 \mathrm{~m}^{2} \mathrm{~s}^{-1}$. These values are in a good agreement with most of the literature data obtained from the measurement of the transformation of the shape of periodic

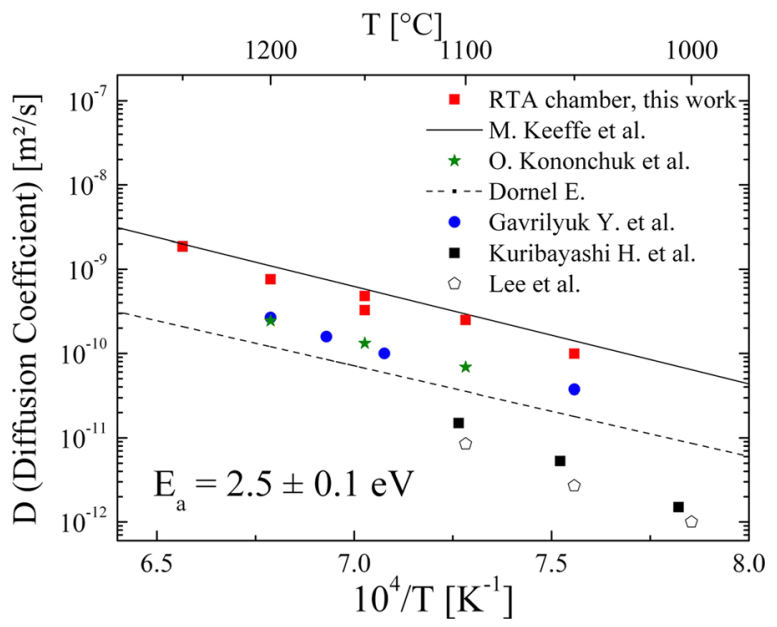

FIG. 9. Surface diffusivity coefficient of crystal silicon (100) obtained from the RTA experiment compared with literature data. ${ }^{5-7,13,20,21}$ superficial silicon structures. ${ }^{7,13,20,21}$ However, both Kuribayashi et al. ${ }^{5}$ and Lee et al. ${ }^{6}$ have reported lower diffusivities with higher activation energies $(E a=3.5 \mathrm{eV})$. We note that their experimental data are obtained using structures with high aspect ratios (high local curvature) where the $\mathrm{M}-\mathrm{H}$ model approximation is not appropriated (see the numerical analysis of the limits of M-H approximation in Ph.D. thesis by Dornel ${ }^{21}$ ). They also studied annealing at much lower temperatures, below the silicon roughening temperature which is confirmed by the observation of terraces on the silicon surfaces. Because of the effects of surface anisotropy and diffusion barriers at the edges of the terrace, the simple M-H surface diffusion model could lead to incorrect extracted values of $D_{s}$ under these conditions.

We have also studied the time and temperature evolution of the stochastic terms that we have introduced. Figure 10 shows the time and temperature evolutions of coefficients of the noise terms $\left(\Delta_{N C N}\right.$ and $\left.\gamma_{C N}\right)$. We do not observe any significant change of the non-conservative noise coefficient $\left(\Delta_{N C N}\right)$ with the annealing temperature. Nevertheless, it can be noted that $\Delta_{N C N}$ decreases with time as $t^{-1 / 2}$. Based on these observations, we can speculate that the non-conservative noise is a result of etching (silicon oxidation followed by oxide desorption) of the silicon surfaces by residual oxygen in the process chamber. Residual oxygen can be consumed by silicon oxidation and purged by the $\mathrm{Ar} / \mathrm{H}_{2}$ flow, thus resulting in a decrease of the noise coefficient over time. ${ }^{22}$

Besides, we can observe in Figure 10 that the CN coefficient $\left(\gamma_{C N}\right)$, which describes the thermal fluctuations of the surface, remains constant over the time. It can be also noted that $\gamma_{C N}$ increases with the annealing temperature. Figure 11 shows this evolution of $\gamma_{C N}$ as a function of the annealing temperature. This evolution can be fitted by an Arrhenius type law with a pre-exponential factor $A \sim 3.9 \times 10^{11} \mathrm{~nm}^{4}$ and an activation energy of $3.13 \mathrm{eV}$. This again indicates a thermal activated phenomenon, in agreement with our proposal to inject in the model some conservative noise describing thermal fluctuations.

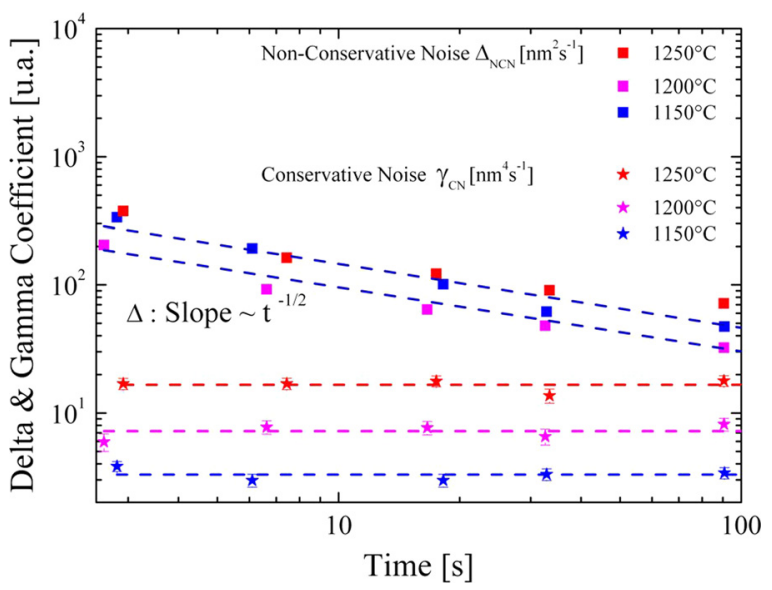

FIG. 10. Plot of the noise coefficients $\Delta_{N C N}$ and $\gamma_{C N}$ (conservative noise and non-conservative, respectively) as a function of annealing time. The annealing temperature was $1200^{\circ} \mathrm{C}$. 


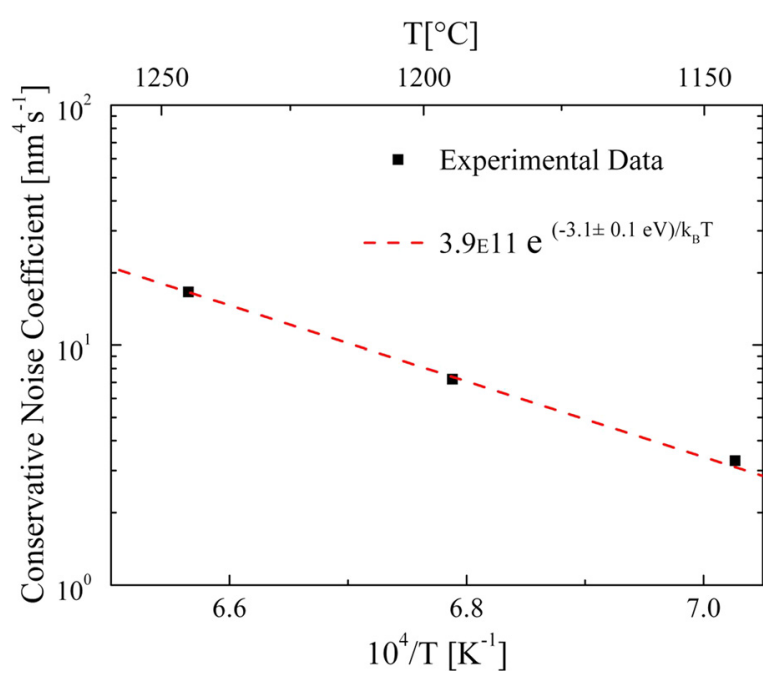

FIG. 11. Arrhenius plot of conservative noise coefficient $(\gamma)$ describing the thermal fluctuations of the system. The activation energy is approximately $3.1 \mathrm{eV}$.

We can estimate the theoretical limit of the smoothening by RTA. In principle, the lowest roughness that could be reached by RTA can be calculated from the limit of Eq. (6) for infinite time. The lowest roughness (corresponding to a $30 \times 30 \mu \mathrm{m}^{2}$ AFM image) that can be obtained by annealing at $1200^{\circ} \mathrm{C}$ in a reducing ambient is estimated to be approximately equal to $R M S=0.75 \AA$. In order to obtain smoother surfaces, one should favor thermal annealing at lower temperatures which limits the contribution of the $\mathrm{CN}$ to the final roughness. Furthermore, if the annealing temperature is lower than the roughening temperature, the annealing leads to a faceted surface which could be atomically flat. ${ }^{12}$ However, a low temperature process is much more time consuming. In addition, an ultra-clean chamber and ultra-pure gas flow could be used in order to reduce the oxygen contamination and then to limit the contribution of the NCN to the surface roughness. $^{23}$

The spectral evolution of the surface topography during a thermal annealing has been considered and investigated using 2D-PSD functions. We can differentiate three spectral regions by their diffusion behaviour. In the first spectral region (frequencies lower than $f_{D}$ ), no change on the amplitude of the 2D-PSD function is observed (see region $I$ in Fig. 6). It means that no diffusion phenomenon take place over this spectral region. In the second region (spatial frequencies between $f_{D}$ and $f_{N}$ ), the reduction of the amplitude of the 2D-PSD function shows a scaling behaviour corresponding to the self-diffusion (see region $I I$ in Fig. 6). It implies that self-diffusion is the predominant diffusion mechanism over this region. Finally, in the third region (spatial frequencies higher than $f_{N}$ ) two phenomena are observed. For spatial frequencies between $f_{N}$ and $f_{C N}$ the oxidation-evaporation mechanism explains the surface topography evolution while for frequencies higher than $f_{C N}$ thermal fluctuation of adatoms explains the evolution of the surface topography (see regions $I I I_{a}$ and $I I I_{b}$ in Fig. 6, respectively).

\section{CONCLUSIONS}

The surface self-diffusion of silicon adatoms on rough SOI wafers was investigated by means of AFM and quantified using 2D-PSD functions. By introducing two stochastic terms, corresponding to conservative and non-conservative noises into the simple $\mathrm{M}-\mathrm{H}$ equation, a parametric model describing the surface evolution during high temperature annealing in reducing atmosphere has been proposed. These stochastic terms describe the surface thermal fluctuations and the oxidation-evaporation phenomenon, respectively. Very good agreement between experimental and theoretical data, describing both the roughness evolution of the surface and the kinetics of surface self-diffusion was obtained. The temperature dependence of the self-diffusivity of silicon follows an Arrhenius-type law with an activation energy of $E a=2.5 \pm 0.1 \mathrm{eV}$ and a pre-exponential factor of approximately $0.3 \mathrm{~m}^{2} \mathrm{~s}^{-1}$. We have also studied the time and temperature evolutions of the parameters describing the stochastic terms we have introduced into the model. Using this optimized model, we can now predict the decrease of each spatial frequency of the 2D-PSD amplitude, over a very large spatial bandwidth. Finally, the limits of the smoothening process by RTA are discussed. Two ways can be used to obtain even smoother surfaces; annealing at lower temperatures (below the roughening temperature) and/or using ultra-pure experimental conditions limiting oxygen contamination of the surface. The pressure and ambient dependences of the parameters describing both the conservative and nonconservative stochastic noises require additional investigation and will be reported in detail in a future work.

\section{ACKNOWLEDGMENTS}

The authors acknowledge useful discussions with Didier Landru and Sebastien Kerdiles from Soitec and Malte Henkel from Institut Jean Lamour. We also would like to thank Elodie Douguet for wafers preparation.

${ }^{1}$ O. Weber, O. Faynot, F. Andrieu, C. Buj-Dufournet et al., "High immunity to threshold voltage variability in undoped ultra-thin FDSOI MOSFETs and its physical understanding," in IEEE International Electron Devices Meeting, IEDM 2008, pp. 1-4.

${ }^{2}$ T. Hook, M. Vinet, R. Murphy, S. Ponoth, and L. Grenouillet, "Transistor matching and silicon thickness variation in ETSOI technology," in IEEE International Electron Devices Meeting (IEDM) (2011), pp. 5.7.1-5.7.4.

${ }^{3}$ P. E. Acosta-Alba, O. Kononchuk, G. Riou, C. Moulin et al., "Multi-scale thickness and roughness characterization of thin silicon-on-insulator films," ECS J. Solid State Sci. Technol. 2, P357 (2013).

${ }^{4}$ A. L. Barabasi and H. E. Stanley, Fractal Concepts In Surface Growth, edited by C. U. Press (Cambridge Press, 1995).

${ }^{5}$ H. Kuribayashi, R. Hiruta, R. Shimizu, K. Sudoh, and H. Iwasaki, "Shape transformation of silicon trenches during hydrogen annealing," J. Vac. Sci. Technol., A 21, 1279 (2003).

${ }^{6}$ M. -C. M. Lee and M. Wu, "Thermal annealing in hydrogen for 3-D profile transformation on silicon-on-insulator and sidewall roughness reduction," J. Microelectromech. Syst. 15, 338-343 (2006).

${ }^{7}$ M. E. Keeffe, C. Umbach, and J. M. Blakely, "Surface self-diffusion on si from the evolution of periodic atomic step arrays," J. Phys. Chem. Solids 55, 965-973 (1994).

${ }^{8}$ W. W. Mullins, "Theory of thermal grooving," J. Appl. Phys. 28, 333 (1957).

${ }^{9}$ C. Herring, "Effect of change of scale on sintering phenomena," J. Appl. Phys. 21, 301 (1950). 
${ }^{10}$ E. van Vroonhoven, H. J. Zandvliet, and B. Poelsema, "A quantitative evaluation of the dimer concentration during the $(2 \times 1)-(1 \times 1)$ phase transition on Ge(001)," Surf. Sci. 574, L23-L28 (2005).

${ }^{11} \mathrm{~V}$. Ignatescu, "Engineering surface morphology at the atomic level with applications in electronic materials," Ph.D. dissertation (Cornell University 2007).

${ }^{12}$ T. Doi, M. Ichikawa, and S. Hosoki, "Observation of Si(001) surface topography at temperatures below $1140{ }^{\circ} \mathrm{C}$ using a reflection electron microscope," Phys. Rev. B 55, 1864-1870 (1997).

${ }^{13}$ Y. L. Gavrilyuk, Y. S. Kaganovjkii, and V. G. Lifshits, "Diffusive mass transfer on the (111) and (100) surfaces of silicon single crystals," Sov. Phys. Crystallogr. 26, 317-322 (1981).

${ }^{14}$ S. Personnic, K. K. Bourdelle, F. Letertre, A. Tauzin et al., "Impact of the transient formation of molecular hydrogen on the microcrack nucleation and evolution in h-implanted si (001)," J. Appl. Phys. 103, 023508 (2008).

${ }^{15}$ X. Li, A. Teramoto, T. Suwa, R. Kuroda, S. Sugawa, and T. Ohmi, "Formation speed of atomically flat surface on si (100) in ultra-pure argon," Microelectron. Eng. 88, 3133-3139 (2011).

${ }^{16}$ H. Liu, W. Zhou, Q.-M. Nie, and Q.-H. Chen, "Depinning transition of the quenched Mullins - Herring equation: A short-time dynamic method," Phys. Lett. A 372, 7077-7080 (2008).
${ }^{17}$ E. Darvish and A. A. Masoudi, "Kinetic surface roughening for the Mullins - Herring equation,” J. Math. Phys. 50, 013304 (2009).

${ }^{18}$ J. M. López, M. A. Rodríguez, and R. Cuerno, "Power spectrum scaling in anomalous kinetic roughening of surfaces," Phys. A 246, 329-347 (1997).

${ }^{19}$ K. A. Takeuchi, M. Sano, T. Sasamoto, and H. Spohn, "Growing interfaces uncover universal fluctuations behind scale invariance," Sci. Rep. 1, 34 (2011).

${ }^{20}$ O. Kononchuk, D. Landru, and C. Veytizou, "Novel trends in SOI technology for CMOS applications," Solid State Phenom. 156-158, 69-76 (2010).

${ }^{21}$ E. Dornel, "Évolution morphologique par diffusion de surface et application à l'étude du démouillage de films minces solides", Ph.D. dissertation (Univertité Joseph Fourier, Grenoble, 2007).

${ }^{22}$ B. Mohadjeri, M. R. Baklanov, E. Kondoh, and K. Maex, "Oxidation and roughening of silicon during annealing in a rapid thermal processing chamber," J. Appl. Phys. 83, 3614 (1998).

${ }^{23}$ R. Kuroda, T. Suwa, A. Teramoto, R. Hasebe, S. Sugawa, and T. Ohmi, "Atomically flat silicon surface and silicon/insulator interface formation technologies for (100) surface orientation large-diameter wafers introducing high performance and low-noise metal-insulator-silicon FETs," IEEE Trans. Electron Devices 56, 291-298 (2009). 